\title{
Discrete time crystal in a finite chain of Rydberg atoms without disorder
}

\author{
Chu-hui Fan $\odot,{ }^{1,{ }^{*}}$ D. Rossini, ${ }^{2, *}$ Han-Xiao Zhang, ${ }^{1}$ Jin-Hui Wu, ${ }^{1, \dagger}$ M. Artoni, ${ }^{3}$ and G. C. La Rocca ${ }^{4, *}$ \\ ${ }^{1}$ School of Physics, Northeast Normal University, Changchun 130024, China \\ ${ }^{2}$ Dipartimento di Fisica, Università di Pisa and Istituto Nazionale di Fisica Nucleare, Largo Pontecorvo 3, I-56127 Pisa, Italy \\ ${ }^{3}$ Department of Engineering and Information Technology and Istituto Nazionale di Ottica (Consiglio Nazionale delle Ricerche), \\ Brescia University, 25133 Brescia, Italy \\ ${ }^{4}$ Scuola Normale Superiore and Consorzio Nazionale Interuniversitario per le Scienze Fisiche della Materia, 56126 Pisa, Italy
}

(Received 7 July 2019; published 13 January 2020)

\begin{abstract}
We study the collective dynamics of a clean Floquet system of cold atoms, numerically simulating two realistic setups based on a regular chain of interacting Rydberg atoms driven by laser fields. In both cases, the population evolution and its Fourier spectrum display clear signatures of a discrete time crystal (DTC), exhibiting the appearance of a robust subharmonic oscillation which persists on a timescale increasing with the chain size, within a certain range of control parameters. We also characterize how the DTC stability is affected by dissipative processes, typically present in this atomic system even though the Rydberg state is very long lived.
\end{abstract}

DOI: 10.1103/PhysRevA.101.013417

\section{INTRODUCTION}

Spontaneous symmetry breaking is essential to modern physics [1]. Inspired by the concept of crystal order in space, in 2012 Wilczek first proposed the idea of a timecrystal phase, corresponding to spontaneous time-translationsymmetry breaking [2], whereby time-periodic properties, i.e., a clock, emerge in a time-invariant dynamical system. This intriguing idea was found to be unfeasible [3,4] at thermal equilibrium, though it can be suitably generalized $[5,6]$. It was soon realized, however, that periodically driven (i.e., Floquet) systems may enter a discrete time-crystal (DTC) phase [7-11], in which the dynamics is governed by a periodicity that is different (typically a subharmonic) from that of the Hamiltonian. Signatures of the DTC phase have been observed in a variety of experimental platforms, e.g., in trapped ions [12], diamond nitrogen vacancy centers [13], superfluid systems $[14,15]$, and spin nuclear magnetic resonance (NMR) systems [16,17]. Meanwhile, many extensions of the DTC paradigm have been theoretically discussed as, e.g., the possibility to stabilize critical time crystals [18], prethermal time crystals $[19,20]$, boundary time crystals [21], Dicke time crystals [22,23], fractional time crystals [24], or even topological time crystals [25,26].

The onset of the nonequilibrium DTC phase implies the self-reorganization of a Floquet many-body system, such that the dynamic behavior of an observable switches to an oscillatory motion characterized by a spontaneously chosen period which differs from that of the Hamiltonian $[27,28]$. In addition to the periodic driving and the many-body interactions, the presence of disorder in the system was also initially assumed

\footnotetext{
*These authors contributed equally to this work.

$\dagger$ jhwu@nenu.edu.cn

"giuseppe.larocca@sns.it
}

to be a prerequisite for the stabilization of the DTC phase, as a consequence of many-body localization [29,30]. However, in view of experimental $[13,17]$ and theoretical $[31,32]$ findings, the occurrence of a DTC phase has remarkably turned out to be viable even in a clean system, i.e., without disorder, and this case has recently attracted a great deal of attention [33-36]. The issue of prethermalization favored by a fast driving, as well as the detrimental or beneficial roles of a dissipative bath, have also been discussed [19,37,38].

In this context, the investigation of the robustness and limitations of the DTC phase in different systems is clearly of value. The progress in manipulating cold atoms has made them a unique setting for the quantum simulation of manybody systems [39], in particular when exploiting Rydberg atom arrays with controllable spatial configurations [40-43]. Rydberg atoms of high principal numbers [44] experience strong dipole-dipole interactions that can be tailored under appropriate level configurations to realize a variety of effects, including dipole blockade [45], dipole antiblockade [46], cooperative nonlinearity $[47,48]$, quantum many-body scars [49], and in-phase or antiphase dynamics [50]. Consequently, Rydberg atoms have been proven to be a promising platform for implementing many quantum tasks such as, e.g., the creation of quantum gates [51-53], the generation of entanglement states [54,55], or the realization of photonic devices [56-58]. Recently, new features of the collective dynamics of Rydberg atoms beyond equilibrium states have been studied under Floquet driving in the presence of disorder [59].

A Floquet system is said to be in the DTC phase when the following three conditions are satisfied [31,32]: (i) Timetranslation symmetry breaking: an observable displays an oscillation with frequency different than that of the driving (typically a lower subharmonic); (ii) rigidity: the spontaneously generated frequency is robust against parameter variations; (iii) persistence: the time over which the oscillation remains in phase increases with the system size (temporal 


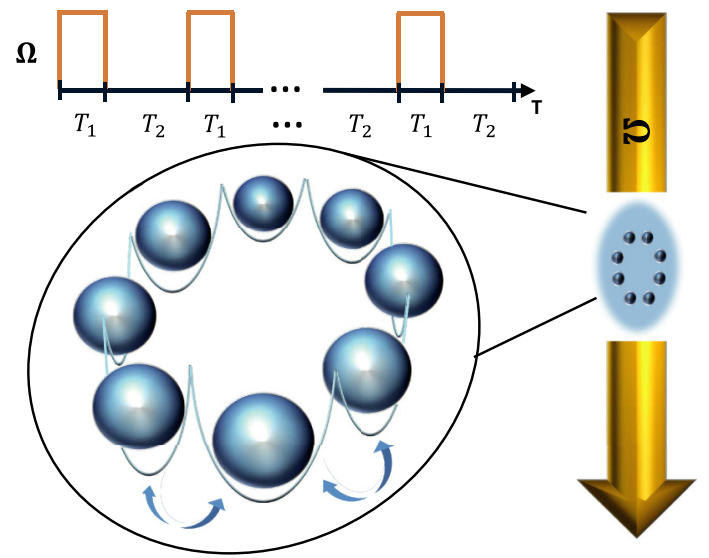

FIG. 1. Schematic chain of eight equally spaced Rydberg atoms trapped in optical potentials. The atoms interact through a nearestneighbor coupling $V$, and are driven by a common laser field of Rabi frequency $\Omega$. Such driving field is periodically switched on $\left(T_{1}\right)$ and off $\left(T_{2}\right)$.

long-range order). Although, in principle, the above conditions should hold in the thermodynamic limit, the consideration of finite systems is definitely relevant to experiments [12], while helping in gaining insight into the onset of the DTC phase.

The aim of the present work is the exact numerical simulation of two realistic setups without disorder, based on a finite chain of cold Rydberg atoms. Such a platform could be implemented in experiments [39-43] and would provide a flexible tuning of Floquet driving and of many-body interactions, accompanied by long coherence times. In particular, as sketched in Fig. 1, the Rydberg atoms, arranged along a ring and coupled via nearest-neighbor dipole-dipole interactions, behave as two-level systems driven by laser fields periodically turned "on" and "off." The first setup we consider requires only one driving responsible for the Rabi cycling between the ground and the Rydberg states, while the second setup includes an extra dressing field allowing to control the relevant transition frequency via a light shift which is also turned on and off at the Floquet frequency.

Both setups can be modeled by spin Hamiltonians [27,28] which turn out to be distinct from others previously used to describe clean DTC systems [31-36]. In particular, the authors of Ref. [31] considered a ladder model in which intrachain and interchain couplings were alternately turned on and off during each Floquet cycle. The authors of Ref. [32] considered an infinite-range Lipkin-Meshkov-Glick model. The authors of Ref. [33] considered an Ising chain in a transverse field under periodic delta kicks. In Ref. [34], the authors considered a semiclassical mean-field approach with a specifically engineered Floquet driving. While in Ref. [35] the authors considered clock models with $n>2$ states. Finally, the authors of Ref. [36] considered an $X X Z$ spin chain mimicking either trapped ions or superconducting qubits. While the phenomenology of the two setups we model is partly similar to that exhibited by other clean DTC systems with a "period doubling" behavior, key features related to experimentally accessible knobs, such as the driving detuning are specific to the realistic Rydberg-atom platform considered here. Indeed, we do not claim that our models are optimized to realize a clean DTC regime, but rather that they are fully appropriate to describe a system of current experimental interest, in which features of a clean DTC should appear under a Floquet driving protocol well within the state-of-the-art of Rydberg-atom spectroscopy.

Our aim is to numerically analyze, without resorting to approximations, the full quantum dynamical behavior of finite chains of increasing length. Thus the results obtained represent bona fide numerical experiments for a specific realistic system, in a regime where analytical insight is lacking despite all the relevant energy scales being comparable. Clearly, the price to pay is the size limitation; we reach, however, sample lengths $(L=14)$ which are at the state-of-the-art in the field of exact numerical approaches [20,22,31-36]. We look for clear signatures of the DTC phase that should persist for sufficiently long times, before other mechanisms such as dissipative processes take over. Our results suggest the onset of a DTC phase, with features and robustness that may depend on the Rabi frequency and the detuning of the driving laser fields, as well as on the interatomic interaction strength. The persistence of a DTC in increasingly larger chains is here characterized as a function of the model parameters. In both of the above setups, although differently sensitive to detuning, definite regularities are unveiled as a function of the model parameters; these can further be understood by resorting to explicit analytical results for a simplified few-cycles and few-bodies regime. Finally, the effects of dissipative processes, which are unavoidable and ultimately due to the decay of the long-lived Rydberg states, are addressed as well.

The paper is organized as follows. We first analyze a system that can be implemented experimentally using a single optical control, describing the model and the basic theoretical approach in Sec. II and discussing the numerical results in Sec. III. We then introduce in Sec. IV a distinct model that, although theoretically simpler, would also require the use of a second optical pump to periodically shift the energy of the relevant atomic transition via the AC Stark effect. We discuss the stability of the DTC phase in increasingly large systems for different parameter ranges in Sec. V and, finally, how it is affected by dissipative processes in Sec. VI. Conclusions are presented in Sec. VII, while the two Appendices are devoted, respectively, to details concerning the numerical simulations and to the simplified analytical treatment.

\section{MODEL AND STROBOSCOPIC EVOLUTION}

We consider a closed chain of $L$ equally spaced cold Rydberg atoms tightly trapped in optical potential wells [40-42], as illustrated in Fig. 1. These atoms can be considered as two-level systems with a common transition frequency $\omega_{g r}$ from their ground $|g\rangle_{j}$ to Rydberg $|r\rangle_{j}$ states, driven at a Rabi frequency $\Omega$. They interact through a nearest-neighbor van der Waals (vdW) coupling of strength $V$, while the driving is alternatively turned on and off in a periodic fashion, so to yield a binary Floquet Hamiltonian $H(t)$ of period $T=T_{1}+T_{2}$, 
whose two components are

$$
\begin{array}{rlrl}
H_{1} & =\hbar \sum_{j=1}^{L}\left[\Omega\left(\sigma_{j}^{+}+\sigma_{j}^{-}\right)+\Delta N_{j}^{r}+V N_{j}^{r} N_{j+1}^{r}\right], & & \text { for } \quad(n-1) T \leqslant t<n T-T_{2}, \quad \text { (first stage) } \\
\text { and } & H_{2}=\hbar \sum_{j=1}^{L}\left(\Delta N_{j}^{r}+V N_{j}^{r} N_{j+1}^{r}\right), & \text { for } \quad n T-T_{2} \leqslant t<n T, \quad \text { (second stage) }
\end{array}
$$

with $n \in\left\{1,2, \ldots, n_{f}\right\}$ and $n_{f}$ marking the final Floquet cycle. Here $\sigma_{j}^{+}=|r\rangle_{j j}\langle g|\left(\sigma_{j}^{-}=|g\rangle_{j j}\langle r|\right)$ refers to the Rydberg transition raising (lowering) operator, while $N_{j}^{r}=|r\rangle_{j j}\langle r|$ $\left(N_{j}^{g}=|g\rangle_{j j}\langle g|\right)$ depicts the Rydberg- (ground-)state projection operator for the $j$ th atom. In the above expressions $\Delta=$ $\omega_{d}-\omega_{g r}$ is the detuning of the driving field; it is worth noting that such detuning also appears in $H_{2}$, even if the driving is turned off in the second stage, because both $H_{1}$ and $H_{2}$ are written in the rotating frame with respect to $\omega_{d}$.

The resulting stroboscopic time evolution of the Rydberg chain described above, after $n$ Floquet cycles, is ruled by the unitary operator

$$
U_{F}(n)=\left[U_{2} U_{1}\right]^{n}=\left[e^{-i H_{2} T_{2} / \hbar} e^{-i H_{1} T_{1} / \hbar}\right]^{n},
$$

where $U_{1} \equiv e^{-i H_{1} T_{1} / \hbar}$ corresponds to the dynamics in the first stage of the Floquet cycle and $U_{2} \equiv e^{-i H_{2} T_{2} / \hbar}$ to that of the second stage. The Floquet operator $U_{F}(n)$ depends on the products $\left\{\Omega T_{1}, \Delta T_{1}, V T_{1}, \Delta T_{2}, V T_{2}\right\}$. For the sake of convenience, we further define $\epsilon$ as a perturbation of Rabi frequency with $\Omega T_{1}=\pi / 2+\epsilon T_{1}$ (the unperturbed value $\Omega T_{1}=\pi / 2$ corresponding to a Rabi flip-flop every two Floquet periods) and hereafter set $T_{1}=1 \mu$ s (i.e., $1 / T_{1}=1 \mathrm{MHz}$ ) thus fixing the unit of time (frequency).

In models describing the onset of DTC signatures [10,31], it is common to turn off the many-body interactions during the first part of the Floquet cycle, i.e., in $H_{1}$, keeping them only in the second part, i.e., in $H_{2}$. This procedure seems unfeasible for a realistic Rydberg-atom platform, as it would require changing the relative distance among atoms rapidly and in a controlled fashion since the interatomic coupling $V$ depends on the interatomic distance. A definitely simpler strategy to reduce the influence of $V$ in $H_{1}$ is to set $\Omega \gg V$ by applying strong-enough driving fields, while setting $T_{1} \ll T_{2}$, to guarantee that $\Omega T_{1} \approx V T_{2}$, such that the effect of the internal many-body interactions is comparable to the one of the external driving responsible for the Rabi flipping.

\section{A. Observables}

As is typically done in the study of DTC phases, we will present our results in terms of three related observables $[10,13,18,31]$, namely the population difference $P$, its Fourier transform $S$, and a binary order parameter $Q$, that serve to assess the onset of a DTC phase. The Rydberg chain atomic population difference (i.e., the average population imbalance between Rydberg and ground states), is defined as

$$
P(n)=\frac{1}{L} \sum_{j=1}^{L}\left\langle\Psi(n)\left|N_{j}\right| \Psi(n)\right\rangle,
$$

where $|\Psi(n)\rangle=U_{F}(n)|\Psi(0)\rangle$ denotes the collective state after $n$ Floquet cycles described above, starting from the initial state $|\Psi(0)\rangle=|g\rangle_{1}|g\rangle_{2} \ldots|g\rangle_{L}$ which is the natural choice for this experimental platform, while $N_{j}=N_{j}^{r}-N_{j}^{g}$ is the population difference operator for the $j$ th atom.

In the case of a large-enough number of Floquet cycles $n_{f} \gg 1$, the DTC features can be also assessed by looking at the population difference of the Fourier spectrum $[10,13]$

$$
S(v)=\frac{1}{n_{f}} \sum_{n=1}^{n_{f}} P(n) \exp (2 \pi i n v),
$$

which provides a simple picture on the time-translationsymmetry breaking, by showing one or more resonant peaks (in the above equation $n_{f}$ is the total number of Floquet cycles, and its inverse stands for a normalization coefficient).

Since $P(n)$ oscillates continuously between positive and negative values with two extreme \pm 1 , to check the stability of the DTC phase, we introduce the binary order parameter [18]

$$
Q(n)=\operatorname{sgn}\left[(-1)^{n} P(n)\right]
$$

With our choice of initial state, in the DTC phase of $2 T$ periodicity, one finds a constant value $Q(1)=Q(2)=\cdots=$ $Q\left(n_{f}\right)=-1$, indicating that the oscillation frequency is fixed during the whole evolution process. Otherwise, $Q(n)= \pm 1$ will appear alternatively for identical or distinct numbers of Floquet cycles, indicating that two or more oscillation frequencies exist before the evolution process stops.

\section{B. Experimental feasibility}

Our model can be realized with present-day cold-atom technology [40-43], in a three-level ladder configuration for ${ }^{87} \mathrm{Rb}$ atoms with, e.g., the Rydberg state $|r\rangle=\mid 60 s, j=1 / 2$, $\left.m_{j}=1 / 2\right\rangle$, the excited state $|e\rangle=\left|5 P_{1 / 2}, F=1, m_{F}=0\right\rangle$, and the ground state $|g\rangle=\left|5 S_{1 / 2}, F=1, m_{F}=1\right\rangle$ $[44,60,61]$. Experimentally, the Rydberg-state $|r\rangle$ is populated from the ground-state $|g\rangle$ via a two-photon transition nearly resonant with the intermediate state $|e\rangle$, but the last state can be adiabatically eliminated in the case of a small two-photon detuning $\Delta$ and two large single-photon detunings, so that the three-level ladder configuration can be effectively described by our two-level model (referring to states $|g\rangle$ and $|r\rangle$, driven by a single generalized Rabi frequency $\Omega$ with the detuning $\Delta)[60,62]$.

The upper Rydberg state $|r\rangle$ has a decay rate $\Gamma \simeq 10 \mathrm{kHz}$ and a vdW coefficient $C_{6} \simeq 9 \times 10^{11} \mathrm{~s}^{-1} \mu \mathrm{m}^{6}$, yielding the vdW potential $V=C_{6} / R^{6} \in\{0.3 \mathrm{MHz}, 0.005 \mathrm{MHz}\}$ for a moderate interatomic distance $R \in\{12 \mu \mathrm{m}, 24 \mu \mathrm{m}\}$ 

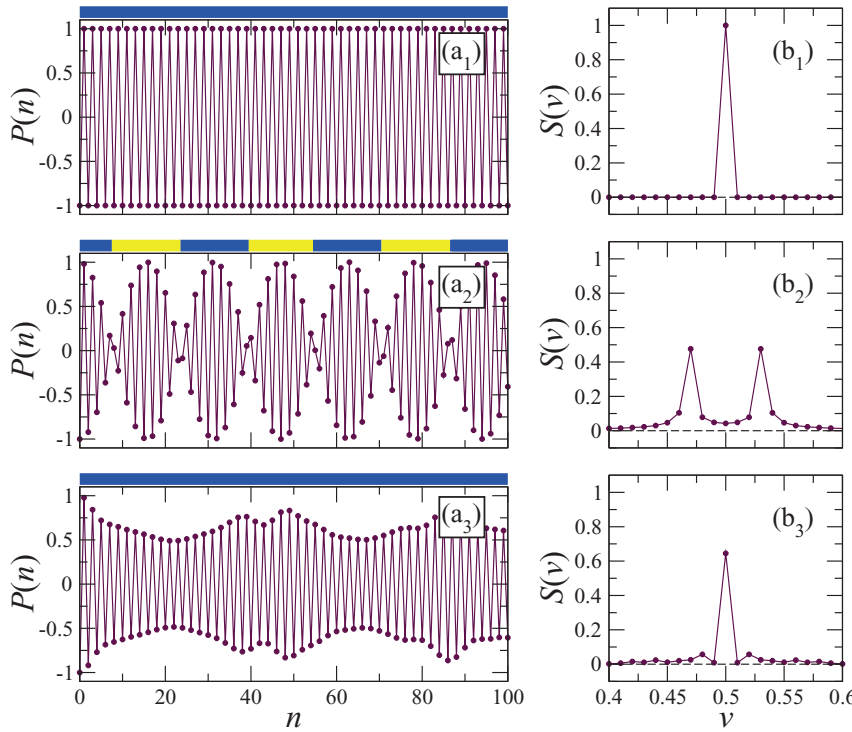

FIG. 2. The average population difference $P(n)$ (left panels) and corresponding Fourier spectra $S(v)$ (right panels) for a closed chain of $L=8$ Rydberg atoms, in the case of zero detuning $(\Delta=0)$. The various panels stand for $\left[\left(\mathrm{a}_{1}\right)\right.$ and $\left.\left(\mathrm{b}_{1}\right)\right] \epsilon=V=0 ;\left[\left(\mathrm{a}_{2}\right)\right.$ and $\left.\left(\mathrm{b}_{2}\right)\right] \epsilon=0.1 \mathrm{MHz}$ and $\left[\left(\mathrm{a}_{3}\right)\right.$ and $\left.\left(\mathrm{b}_{3}\right)\right] V=0 ; \epsilon=V=0.1 \mathrm{MHz}$, and $T_{2}=10 \mu \mathrm{s}$. Here and in the next figures, the order parameter $Q(n)$ is plotted in colorbars over the left panels, with $Q(n)=-1$ and $Q(n)=1$ shown in blue and yellow, respectively.

[44,45,61]. In the following, we mostly take into account only nearest-neighbor atom-atom interactions with $V \approx 0.1 \mathrm{MHz}$, and initially disregard all dissipative effects. However, since the decay rate of the Rydberg state introduces an unavoidable source of dissipation, its consequences will be considered in Sec. VI to assess realistic limits for our simulations.

\section{RESULTS}

Let us now present the outcomes of some numerically exact calculations, aimed at revealing whether our model of Rydberg chain, described by the stroboscopic time evolution of the Hamiltonian in Eqs. (1) and (2), can exhibit the typical DTC features (see also Appendix A for details). Specifically, we shall consider $\epsilon$ and $\Delta$ as two kinds of perturbation in the presence of a nonvanishing $V$, and examine their effects on the population difference $P(n)$, its Fourier spectrum $S(v)$, and the corresponding order parameter $Q(n)$. A qualitative insight on some of our findings can also be gained through a direct analysis of the few-cycle dynamics for two and three atoms [the corresponding analytic expressions of $P(n)$ being provided in Appendix B].

We start by taking $\Delta=0$ in Fig. 2. For $\epsilon=0$ and $V=0$ [Figs. $2\left(a_{1}\right)$ and $\left.2\left(b_{1}\right)\right]$, the atomic polarization trivially exhibits perfect Rabi flips between $P(n)= \pm 1$ and $P(n+1)=$ $\mp 1$ in any two consecutive Floquet cycles, indicating a temporal periodicity which is twice as that of $H(t)$, and further verified by an invariant order parameter $Q(n)=-1$ and a single subharmonic peak at $v=0.5$ in $S(v)$. Since $Q$ is a binary variable, in all the figures we represent it pictorially with a blue or yellow colorbar over the corresponding
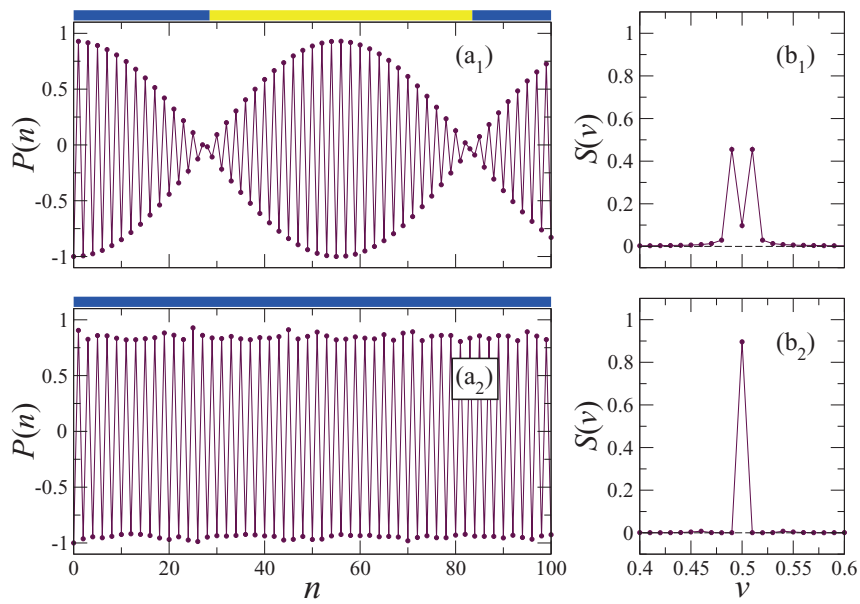

FIG. 3. Same as in Fig. 2, but for $\Delta=0.6 \mathrm{MHz}$ and in the case of zero perturbation of the Rabi frequency $\epsilon=0$ (i.e., $\Omega T_{1}=\pi / 2$ ). Panels $\left(\mathrm{a}_{1}\right)$ and $\left(\mathrm{b}_{1}\right)$ are for $V=0$, while panels $\left(\mathrm{a}_{2}\right)$ and $\left(\mathrm{b}_{2}\right)$ are for $V=0.1 \mathrm{MHz}$ and $T_{2}=10 \mu \mathrm{s}$.

panels. The results become very different in the presence of a nonzero $\epsilon$ [Figs. $2\left(\mathrm{a}_{2}\right)$ and $\left.2\left(\mathrm{~b}_{2}\right)\right]$ : the perturbation $\epsilon$ indeed results in an excessive or inadequate rotation, so that $P(n)$ exhibits beating-like oscillations of period $n_{b}=\pi /(2|\epsilon|)$ (in the above panels we fixed $\epsilon=0.1$, and thus $n_{b} \approx 16$ ). As a consequence, $Q(n)$ periodically switches between -1 and 1 , while $S(v)$ displays two symmetric subharmonic peaks around $v=0.5$. The population imbalance $P(n)$ no longer exhibits a period twice as that of $H(t)$, and the perfect temporal periodicity is lost. Most interestingly, by taking nonzero values both for $\epsilon$ and for $V$, it is possible to recover results that closely resemble those attained for $\epsilon=V=0$. Figures $2\left({ }_{3}\right)$ and $2\left(\mathrm{~b}_{3}\right)$ (where we set $\epsilon=V=0.1 \mathrm{MHz}$ and $T_{2}=10 \mu \mathrm{s}$ ) indeed demonstrate this feature, although the specific value reached by $P(n)$, generally differing from \pm 1 , now becomes $n$ dependent. The corresponding Fourier spectrum acquires two small branch peaks around the previous high central one. This behavior is a signature of a DTC regime induced by the presence of many-body interactions, the period $2 T$ being robust against variations of $\epsilon$.

We proceed by taking a finite detuning $\Delta$ in Fig. 3. Notice, however, that in our case $\Delta$ is always kept uniform and does not introduce any disorder in the system. Figures $3\left(a_{1}\right)$ and $3\left(b_{1}\right)$ show that, in the presence of a detuning $\Delta=0.6 \mathrm{MHz}$ and switching off the $\mathrm{vdW}$ coupling $V$, the atomic polarization exhibits beating-like oscillations, as expected for independent atoms. Each beating period can be estimated as the number $n_{b}$ of Floquet cycles that are needed to recover the same values for any observable in the perfect system $(\Delta=0)$ and in the detuning-perturbed system. Defining the effective Rabi frequency for the perturbed system $\Omega_{e} \equiv \sqrt{\Omega^{2}+\Delta^{2}}$, the above condition is thus enforced by requiring $n_{b} \Omega_{e} T_{1}=$ $n_{b} \Omega T_{1}+2 \pi$, which corresponds to $n_{b} \approx 57$ cycles for $\Delta=$ $0.6 \mathrm{MHz}$ [see the length of the yellow colorbar in Fig. 3( $\left.\mathrm{a}_{1}\right)$ ]. Accordingly, $Q(n)$ periodically switches between its extremal values \pm 1 , and $S(v)$ displays two symmetric peaks around $v=0.5$. Similarly to what observed in Figs. $2\left(a_{3}\right)$ and $2\left(b_{3}\right)$, even in this case we find that vdW interactions are able to 

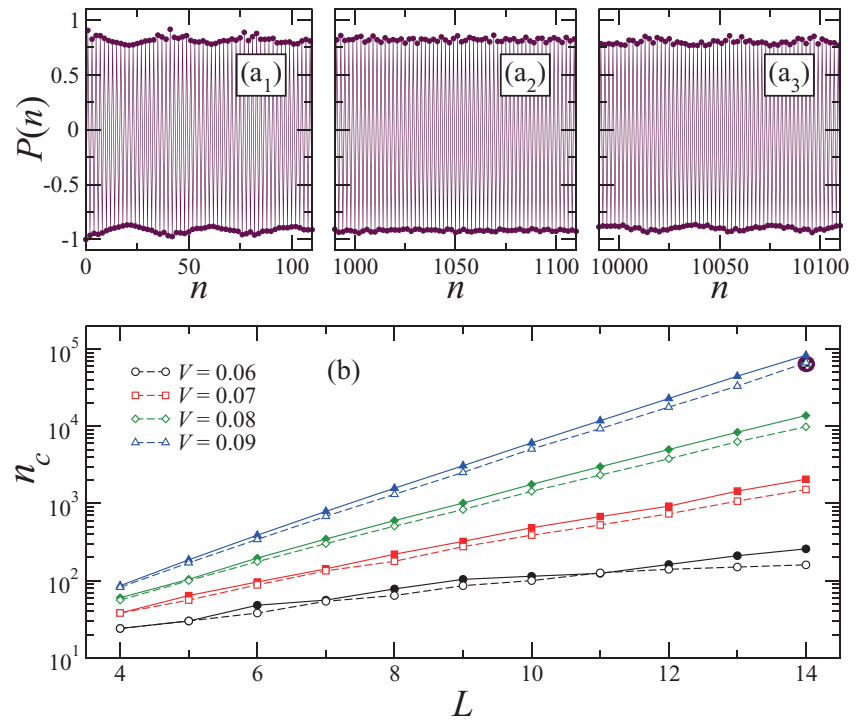

FIG. 4. $\left(a_{1}\right)-\left(a_{3}\right)$ : The average population difference in various Floquet time intervals, for a closed chain of $L=14$ Rydberg atoms with $V=0.09 \mathrm{MHz}$. (b) The critical oscillation number $n_{c}$ against the size $L$ in a semilog scale for different values of $V$; we show here both data with only nearest-neighbor interactions (dashed lines with empty symbols) and including next-nearest-neighbor (n.n.n.) interactions (solid lines with full symbols). The datapoint evidenced with a dark circle indicates the situation highlighted in the three upper panels. The other parameters are chosen as $\Delta=0.6 \mathrm{MHz}$, $\epsilon=0$, and $T_{2}=15 \mu \mathrm{s}$.

stabilize the DTC regime, the period $2 T$ being robust against variations of $\Delta$. This is explicitly shown in Figs. 3( $\left.\mathrm{a}_{2}\right)$ and $3\left(\mathrm{~b}_{2}\right)$, for $V=0.1 \mathrm{MHz}$ and $T_{2}=10 \mu \mathrm{s}$.

We now tackle the issue of persistence, which is one of the distinguishing properties of a DTC phase with increasing system size. Within a certain range of parameters and for a sufficiently large value of $L$, it is possible to observe a remarkable stability of the oscillations of $P(n)$ over tens of thousands of Floquet cycles. This is the case for the situation presented in Figs. 4( $\left.a_{1}\right)$ to $4\left(a_{3}\right)$, where we simulated a chain with $L=14$ Rydberg atoms. We may conclude that, in such a situation, all the three typical DTC features can be observed in a finite interacting Rydberg chain, no matter how its many-body Hamiltonian is perturbed through the Rabi frequency $\epsilon$ or through the detuning $\Delta$. To be more accurate, a scaling analysis of the persistence of the DTC with the system size $L$ should be performed. In this respect a useful quantity is the critical Floquet oscillation number $n_{c}$, which can be defined as the total number of Floquet cycles before $Q(n)$ first changes its sign. This corresponds to the situation in which $Q(n)$ first switches from -1 to +1 (i.e., the smallest value of $n$ for which the colorbar changes color from blue to yellow). Figure 4(b) shows that $n_{c}$ increases exponentially with $L$, at a rate which is sensitive to the strength $V$ of the vdW potential. It is remarkable that, already with a relatively small number of atoms (of the order of ten), a sufficiently strong vdW coupling can stabilize a DTC phase over several thousands of Floquet cycles, thus allowing for a direct experimental test even for a moderate number of
Rydberg atoms. Furthermore, we here considered also the case in which next-nearest neighbor (n.n.n.) interactions are included as well, with $V_{n . n . n .}=V / 2^{6} \simeq 0.016 \mathrm{~V}$; as shown in Fig. 4(b), only minor quantitative changes are evident and, in any case, the presence of n.n.n. interactions is not detrimental to the observation of DTC features.

We finally explore the dependence of the DTC phase on both the detuning $\Delta$ and a nonzero Rabi frequency perturbation $\epsilon$. Figures $5\left(c_{1}\right)$ and $5\left(c_{2}\right)$ reveal a number of interesting features that we briefly summarize here. First of all, the critical oscillation number $n_{c}$ exhibits discrete sharp peaks at specific regularly spaced detunings with amplitudes decaying relatively fast for increasing $|\Delta|$. Second, we notice that the spacing and the amplitude of these peaks are somewhat different for positive [Fig. 5( $\left.\mathrm{c}_{1}\right)$ ] and for negative [Fig. 5( $\left.\mathrm{c}_{2}\right)$ ] perturbations of the Rabi frequency; in particular, the central peaks are higher for positive $\epsilon$. While the figure displays data for $|\epsilon|=0.4 \mathrm{MHz}$, we checked that a qualitatively analogous trend can be found for generic values of $|\epsilon|$. Figures $5\left(a_{1}\right)$ and $5\left(\mathrm{a}_{2}\right)$ and Figs. $5\left(\mathrm{~b}_{1}\right)$ and $5\left(\mathrm{~b}_{2}\right)$ further show that $P(n)$ maintains stable oscillations, although with fast amplitude fluctuations that usually get suppressed with increasing $L$ (not shown). Finally, values of $n_{c}$ attained with a positive $V$ [green data sets in Figs. 5( $\left.c_{1}\right)$ and $5\left(c_{2}\right)$ ] and those attained with a negative $V$ [red data sets in Figs. 5(c $c_{1}$ and $5\left(c_{2}\right]$ display an exact symmetry with respect to $\Delta=0$, indicating invariant results for the simultaneous sign change of $V$ and $\Delta$. Such symmetry holds for any finite values of $V$ and $\Delta$, and can be understood, as discussed in Appendix B, via some analytical insight on the few-cycle dynamics for two and three atoms.

\section{IMPROVED MODEL WITH AN EXTERNALLY CONTROLLED DETUNING}

In the previous section we found that the chain of Rydberg atoms introduced in Sec. II is able to stabilize a DTC phase, with a critical oscillation number $n_{c}$ which is rather sensitive to the detuning. Indeed, as shown in Figs. 5( $c_{1}$ and $5\left(c_{2}\right)$, a small deviation of $\Delta$ from the optimal value may result in a large reduction of $n_{c}$ (as the peaks are quite narrow). It would be desirable to find a practical way to quench such sensitivity which may hinder the detection of DTC signatures.

To this purpose, we now propose an alternative model to that defined in Eqs. (1) and (2). Specifically, one may choose to compensate the detuning $\Delta$ appearing in the rotating frame expression of $\mathrm{H}_{2}$ through the AC Stark effect (or AutlerTownes effect), by turning on in the second stage of the Floquet cycle only, i.e., for $n T-T_{2} \leqslant t<n T$, an additional optical (or microwave) pump which dresses the atom coupling the ground level $|g\rangle$ (or the Rydberg level $\left|r^{\prime}\right\rangle$ ) to an auxiliary excited level $\left|e^{\prime}\right\rangle$ [63-66]. This additional pump can be red or blue detuned, the corresponding light shift compensating for $\Delta$ of either positive or negative sign, with a detuning absolute value large enough to allow for the adiabatic elimination of level $\left|e^{\prime}\right\rangle$. In such case, the Hamiltonian of Eq. (2) simplifies to

$$
\tilde{H}_{2}=\hbar \sum_{j=1}^{L} V N_{j}^{r} N_{j+1}^{r} .
$$



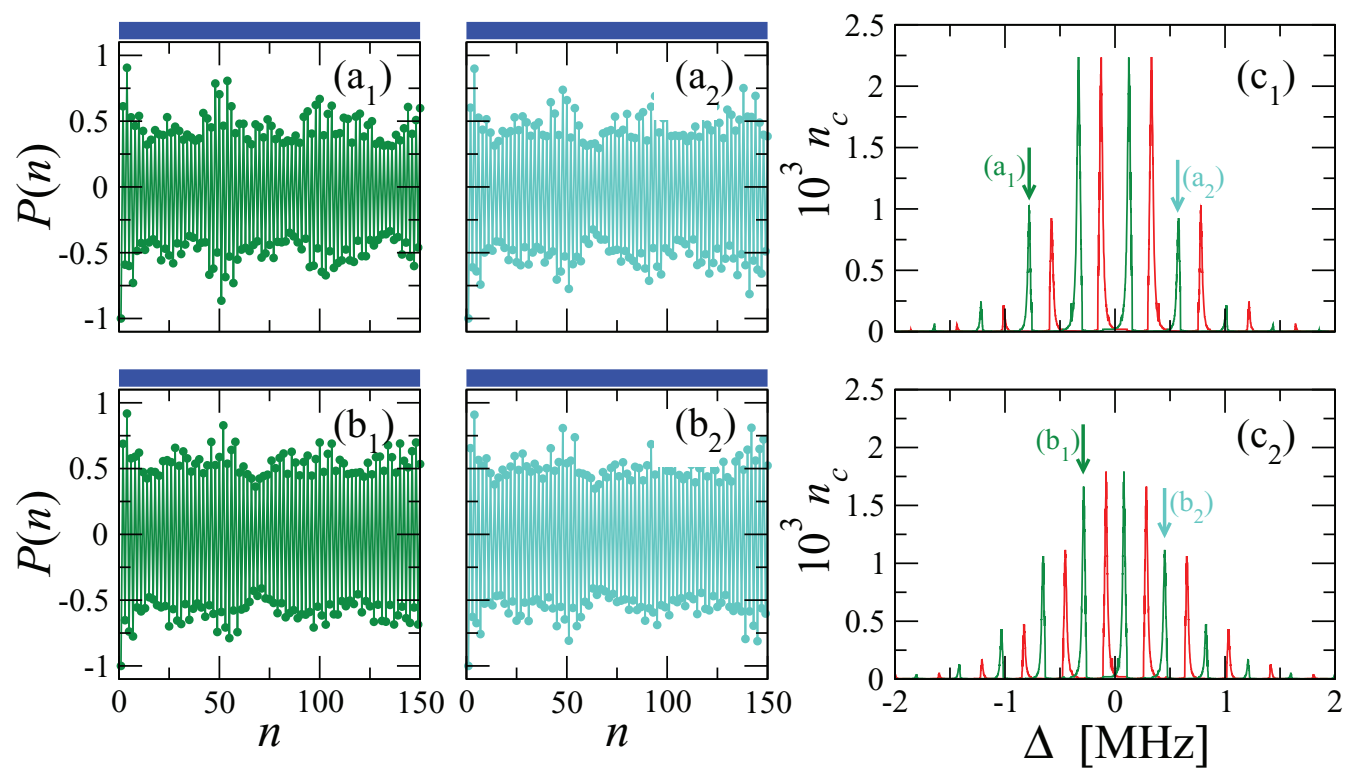

FIG. 5. The average population difference and the critical oscillation number for different values of $\epsilon, \Delta$, and $V$. The three upper panels are for $\epsilon=0.4 \mathrm{MHz}$, while the three lower panels are for $\epsilon=-0.4 \mathrm{MHz}$. $\left(\mathrm{a}_{1}\right)-\left(\mathrm{a}_{2}\right)$ The population difference $P(n)$ for $\Delta=-0.782 \mathrm{MHz}$ and $\Delta=0.578 \mathrm{MHz}$, respectively, as indicated by arrows in panel $\left(\mathrm{c}_{1}\right) .\left(\mathrm{b}_{1}\right)-\left(\mathrm{b}_{2}\right)$ : The population difference $P(n)$ for $\Delta=-0.285 \mathrm{MHz}$ and $\Delta=0.452 \mathrm{MHz}$, respectively, as indicated by arrows in panel $\left(\mathrm{c}_{2}\right) \cdot\left(\mathrm{c}_{1}\right)-\left(\mathrm{c}_{2}\right)$ : The critical oscillation number $n_{c}$ as a function of the detuning $\Delta$. Green data sets stand for $V=0.1 \mathrm{MHz}$, as is the case for all the four panels on the left (arrows point to specific values of $\Delta$ on the green data sets). The red data sets stand for $V=-0.1 \mathrm{MHz}$. In this figure we consider a chain of $L=8$ atoms and fixed $T_{2}=15 \mu \mathrm{s}$.

The new stroboscopic time evolution of this model is thus given by the unitary operator $\widetilde{U}_{F}(n)=$ $\left[\exp \left(-i \tilde{H}_{2} T_{2} / \hbar\right) \exp \left(-i H_{1} T_{1} / \hbar\right)\right]^{n}$. We will refer to this as the improved model, as compared to the original model discussed previously in Sec. II. Since the two models are identical in the case of $\Delta=0$, we examine the effect of a nonzero detuning $\Delta$, now present only in $H_{1}$, on the critical value $n_{c}$.

Figure 6, plotted in the same way as Fig. 4, shows that, also in this alternative model, the DTC features can be stabilized over several thousands of Floquet cycles. Indeed, Figs. 6( $\left.\mathrm{a}_{1}\right)$ to $6\left(\mathrm{a}_{3}\right)$ display the time traces of the population imbalance $P(n)$ in three windows with timescales differing by orders of magnitude, for $L=14$ and $\Delta=0.6 \mathrm{MHz}$. From there, we can see that fixed-frequency oscillations of $P(n)$ persist over a very long time with smooth amplitude fluctuations, which are only slightly suppressed at large times. The critical oscillation number $n_{c}$ also increases exponentially as the chain size $L$ grows, and, compared to the original model, is much less sensitive to the vdW potential $V$ [compare Figs. 4(b) and 6(b)]. Furthermore, even in this improved model, the presence of n.n.n. interactions hardly affects the results and is not detrimental to the observation of DTC features.

Let us now discuss more in detail the effects of the detuning $\Delta$ on the rigidity to perturbations of the DTC phase. To this aim, it is instructive to have a look at Figs. $7\left(\mathrm{c}_{1}\right)$ and $7\left(\mathrm{c}_{2}\right)$, plotted in the same way as the corresponding panels in Fig. 5. From there we observe that $n_{c}$ still exhibits an exact symmetry with respect to $\Delta=0$ for two opposite values of $V$ (as expected again from the analytical results discussed in Appendix B). However, in contrast to the model of Sec. II, the dependence of $n_{c}$ on $\Delta$ yields a smooth envelope rather than distinct sharp peaks. It is also worth noting that such a smooth envelope becomes narrower and higher if the sign of $\epsilon$ is changed from positive [Fig. 7( $\left.\mathrm{c}_{1}\right)$ ] to negative [Fig. 7( $\left.\left.\mathrm{c}_{2}\right)\right]$. This is because the perturbation due to $\Delta$, here present only in $H_{1}$, can be partly suppressed (enhanced) by a negative (positive) $\epsilon$ in the expression for $\Omega_{e}=\sqrt{\left[\pi /\left(2 T_{1}\right)+\epsilon\right]^{2}+\Delta^{2}}$, so that the DTC phase becomes more (less) stable. However, even for this alternative model, the values of $\Delta$ and $\epsilon$ also affect the role of the many-body interactions as the latter depend on the population of the Rydberg level, and there
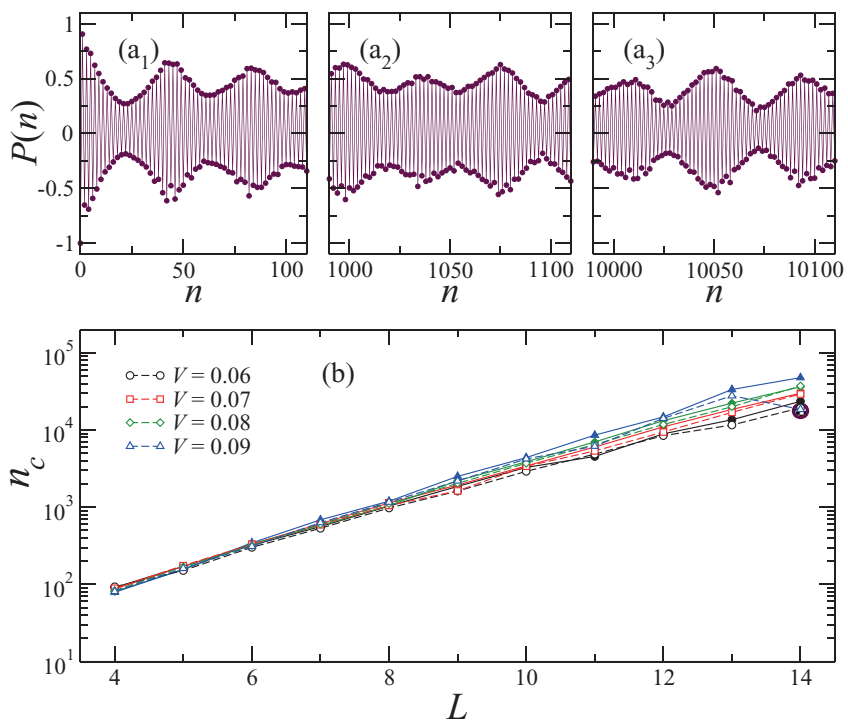

FIG. 6. Same as in Fig. 4, but for the alternative model that compensates the term in $\Delta$ appearing in Eq. (2) [cf., Eq. (7)]. All the various parameters are set as in Fig. 4 . Notice the reduced sensitivity of $n_{c}$ to the vdW potential $V$, as compared to the previous model of Sec. II. 

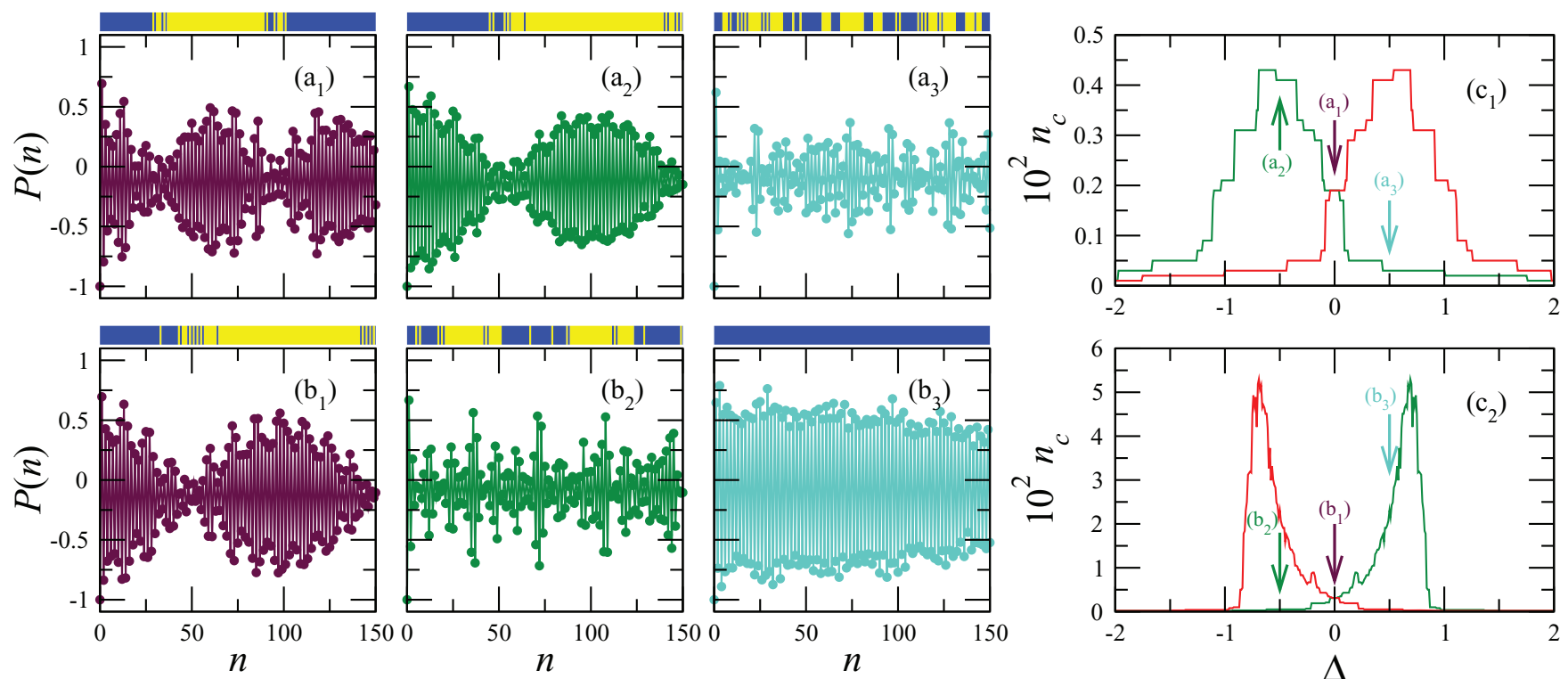

FIG. 7. Same as in Fig. 5, but for the improved Rydberg-atom model [cf., Sec. IV]. Upper panels are for $\epsilon=0.4 \mathrm{MHz}$, while lower panels are for $\epsilon=-0.4 \mathrm{MHz}$. Panels $\left(\mathrm{a}_{1}\right)$ and $\left(\mathrm{b}_{1}\right)$ are for $\Delta=0 \mathrm{MHz}$; panels $\left(\mathrm{a}_{2}\right)$ and $\left(\mathrm{b}_{2}\right)$ are for $\Delta=-0.5 \mathrm{MHz}$; panels $\left(\mathrm{a}_{3}\right)$ and $\left(\mathrm{b}_{3}\right)$ are for $\Delta=0.5 \mathrm{MHz}$. All the plots with $P(n)$ correspond to specific points in the green curves of panels $\left(\mathrm{c}_{1}\right)$ and $\left(\mathrm{c}_{2}\right)$ indicated by arrows. Green data sets in panels $\left(\mathrm{c}_{1}\right)$ and $\left(\mathrm{c}_{2}\right)$ stand for $V=0.1 \mathrm{MHz}$; red data sets stand for $V=-0.1 \mathrm{MHz}$. In this figure we consider a chain of $L=8$ atoms and fixed $T_{2}=15 \mu \mathrm{s}$.

is a complex interplay among $\Delta, \epsilon$ and $V$. Panels $\left(\mathrm{a}_{1}\right)-\left(\mathrm{a}_{3}\right)$ [resp. $\left(\mathrm{b}_{1}\right)-\left(\mathrm{b}_{3}\right)$ ] further show $P(n)$ for three points at different positions of the smooth envelope in panel $\left(c_{1}\right)$ [resp. $\left(c_{2}\right)$ ], from which we can see that a larger $n_{c}$ is always accompanied by more stable oscillations.

All in all, the DTC signatures may be more accessible in experiments implementing the improved model introduced in this section, owing to the reduced sensitivity on $\Delta$. Clearly, this would require a suitable choice of the additional dressing laser field in the second stage of the Floquet cycle.

\section{PARAMETER SPACE ANALYSIS}

As mentioned in the Introduction, the DTC features should be assessed in the thermodynamic limit $L \rightarrow \infty$, while our numerical simulations are limited to finite-size chains (yet of experimental relevance). However, the parameter ranges within which $n_{c}$ increases exponentially with $L$ [as those in Figs. 4(b) and 6(b)] may represent a genuine DTC regime.

A different question regards the dependence of the DTC persistence on the parameter range, as another signature of the DTC regime is its rigidity, i.e., the robustness against parameter variations. Indeed in Figs. 4(b) and 6(b) we have $\Delta \neq 0$ and fixed $\epsilon=0$; in such a case, the exponential increase of $n_{c}$ with $L$ is numerically verified (at least for the sizes we were able to address numerically). However, we found a quite robust numerical evidence that, for $\epsilon \neq 0$, this conclusion holds true only within certain ranges of $\Delta$ values. Incidentally we note that such ranges of parameters are more restrictive for the model of Sec. II, rather than those for the model of Sec. IV. Below we comment on this rather delicate issue.
As a matter of fact, in Fig. 8(a) we explicitly show what happens to the persistence of the DTC regime for the model of Sec. II, by focusing on several $\Delta$ values close to the central peak at $\Delta=0.13 \mathrm{MHz}$ in Fig. $5\left(\mathrm{c}_{1}\right)$ with $\epsilon=0.4 \mathrm{MHz}$. It is clear that the exponential growth of $n_{c}$ does not continue indefinitely, and stable subharmonic oscillations do not survive beyond a critical chain size $L_{c}$, which becomes smaller for a larger deviation from the central peak, consistently with Fig. 5( $\left.\mathrm{c}_{1}\right)$.

To gain further insight on the range of parameters that are amenable to the observation of the DTC features, we now assume that the increase of $n_{c}$ with $L$ being a fair numerical indicator of a putative DTC phase. Bearing in mind this heuristic criterion, we numerically calculated the discrepancy $\delta n_{c} \equiv n_{c}(L)-n_{c}(L-1)$ for a given set of parameters, and identified each point in the parameter space at a given size $L$ that results in $\delta n_{c}>0$ as belonging to the DTC regime. The resulting finite-system phase diagram is shown in the bottom panels of Fig. 8, against the chain size $L$ and the perturbation $\epsilon$. The three panels correspond to, respectively, the simplified model in Appendix B $\left(V=0\right.$ in $H_{1}$, and $\left.\Delta=0\right)$ [Fig. 8(b $\left.b_{1}\right)$, the alternative model of Sec. IV [Fig. $8\left(b_{2}\right]$, and the original model of Sec. II [Fig. 8(b $\left.b_{3}\right)$. It is easy to see that all the models exhibit a boundary delimiting the parameter range which supports a persistent DTC regime (yellow region, characterized by $\delta n_{c}>0$ ) from the rest (green and blue regions, respectively, characterized by $\delta n_{c}=0$ and $\delta n_{c}<0$ ), with the DTC regime being favored for $|\epsilon|$ small enough. Including $V$ in $H_{1}$ and $\Delta$ in $H_{2}$ results in a moderate translation and/or a reduction of the DTC regime along the $\epsilon$ axis, while introducing more frequent instances of a nonmonotonic increase of $n_{c}$ 

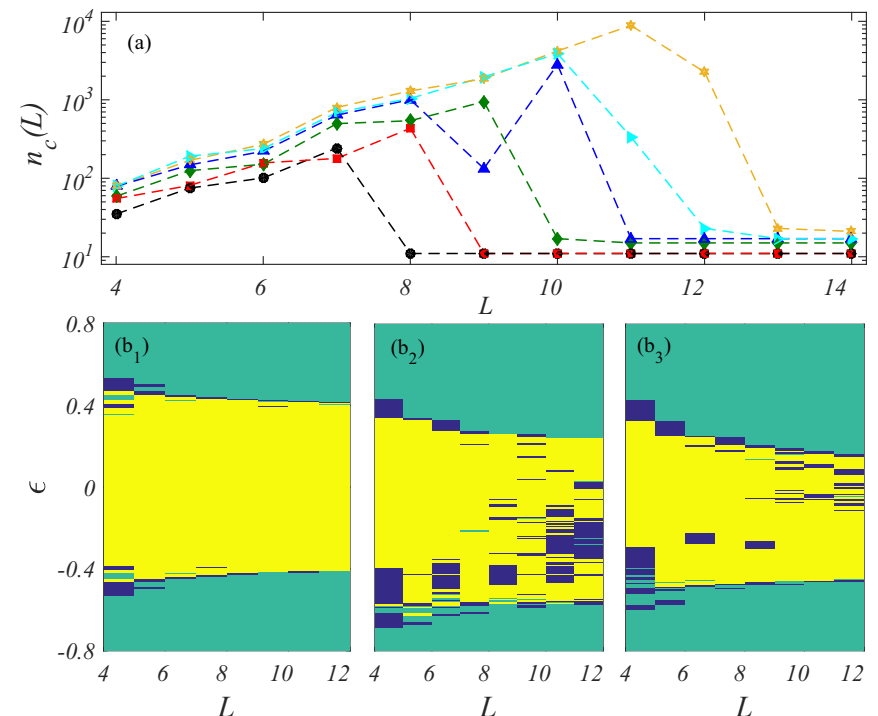

FIG. 8. (a) The critical oscillation number $n_{c}$ against the chain size $L$, for the model of Sec. II with $\Delta=0.16 \mathrm{MHz}$ (black circles), $0.155 \mathrm{MHz}$ (red squares), $0.15 \mathrm{MHz}$ (green diamonds), $0.145 \mathrm{MHz}$ (blue triangles up), $0.143 \mathrm{MHz}$ (cyan triangles right), and $0.142 \mathrm{MHz}$ (orange stars), respectively. The other parameters are chosen as $\epsilon=0.4 \mathrm{MHz}, V=0.1 \mathrm{MHz}$, and $T_{2}=15 \mu \mathrm{s}$. $\left(\mathrm{b}_{1}\right)-\left(\mathrm{b}_{3}\right)$ Finite-size phase diagram of the simplified model in Appendix B $\left[\left(\mathrm{b}_{1}\right) \Delta=0\right.$, $V=0$ in $H_{1}$, and $V=0.1 \mathrm{MHz}$ in $H_{2}$ ], the alternative model of Sec. IV $\left[\left(\mathrm{b}_{2}\right) \Delta=0.8 \mathrm{MHz}\right.$ in $H_{1}, \Delta=0$ in $H_{2}$, and $V=0.1 \mathrm{MHz}$, and the original model of Sec. II [ $\left(\mathrm{b}_{3}\right) \Delta=0.8 \mathrm{MHz}$ and $V=$ $0.1 \mathrm{MHz}$ ], plotted against the chain size $L$ and the perturbation $\epsilon$. The yellow, green, and blue regions, respectively, correspond to $\delta n_{c}>0$, $\delta n_{c}=0$, and $\delta n_{c}<0$.

with $L_{c}$ (blue regions), without, however, changing the overall picture.

Since in all our simulations the energy scale dictated by the Floquet frequency $2 \pi / T$ of the external driving is comparable to the intrinsic energy scales, and, in particular, to the scale $V$ of the many-body interactions, we consider unlikely that the numerical evidence of DTC signatures extending to more than ten thousand cycles [as in Figs. 4( $\left.a_{3}\right)$ and $6\left(a_{3}\right)$ ] might be due to a prethermal time crystal, even though we cannot completely rule out this possibility. Were this to be the case, we might attribute the reduction of the favorable $\epsilon$ range [yellow regions in Figs. $8\left(b_{1}\right)$ to $8\left(b_{3}\right)$ ] to a faster final thermalization in longer chains, preempting the increase of $n_{c}$ with $L$.

\section{DISSIPATION EFFECTS}

While the models we are interested in are amenable to a direct implementation in the laboratory, and the range of chain sizes we numerically simulate $(L \sim 10)$ are clearly of experimental interest, so far we disregarded the fact that the Rydberg state has a long, but finite, lifetime that will unavoidably introduce a source of dissipation in the system. To assess how the DTC features discussed above are possibly affected by the decay rate $\Gamma$ of the Rydberg state $|r\rangle_{j}$ of each atom, we resort to a Markovian master equation treatment [67], describing the time evolution of the density matrix $\rho$ of
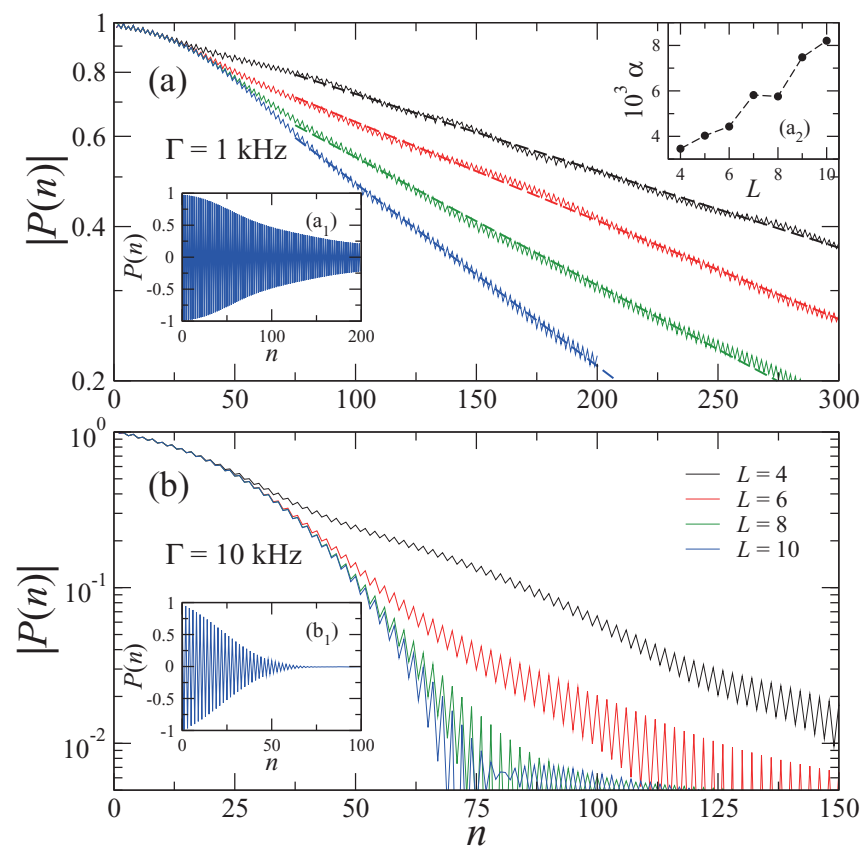

FIG. 9. The modulus of the average population imbalance $|P(n)|$ against the Floquet cycle number $n$ in semilog scale, with (a) $\Gamma=$ $1.0 \mathrm{kHz}$ and (b) $\Gamma=10 \mathrm{kHz}$. Black, red, green, and blue lines correspond to $L=4, L=6, L=8$, and $L=10$, respectively. The other parameters are chosen as $\epsilon=-0.1 \mathrm{MHz}, V=0.1 \mathrm{MHz}, \Delta=0$, $T_{1}=1.0 \mu \mathrm{s}$, and $T_{2}=15 \mu \mathrm{s}$. The two left insets of $\left(\mathrm{a}_{1}\right)$ and $\left(\mathrm{b}_{1}\right)$ show the same time traces of the corresponding main frames for $L=10$, without taking the modulus of $P(n)$. The right inset of $\left(\mathrm{a}_{2}\right)$ shows the decay rate of the population imbalance as a function of $L$, obtained from an exponential fit $|P(n)| \sim e^{-\alpha n}$ (see dashed lines for $n>75$ ) of the numerical data for (a) $\Gamma=1.0 \mathrm{kHz}$.

the $L$-atom system in the Lindblad form

$$
\partial_{t} \rho=-\frac{i}{\hbar}[H(t), \rho]+\mathcal{D}(\rho) .
$$

The incoherent dissipation is described by the term

$$
\mathcal{D}(\rho)=\Gamma \sum_{j=1}^{L}\left[\sigma_{j}^{-} \rho \sigma_{j}^{+}-\frac{1}{2}\left(\rho \sigma_{j}^{+} \sigma_{j}^{-}+\sigma_{j}^{+} \sigma_{j}^{-} \rho\right)\right]
$$

associated with the jump operators $L_{j}=\sqrt{\Gamma} \sigma_{j}^{-}$, describing dissipation processes. Once $\rho(t)$ is attained for our Rydberg chain (see Appendix A for details), it is then straightforward to calculate the population imbalance as $P(t)=\frac{1}{L} \sum_{j=1}^{L} \operatorname{Tr}\left[\rho(n T) N_{j}\right]$.

The outcomes of our simulations for $\Delta=0$ are reported in Fig. 9, where we show a few time traces of the polarization amplitude $|P(n)|$ for different values of the chain size $L$, in the presence of a nonzero $\Gamma$, by directly solving Eq. (8). It is easy to see that, though $|P(n)|$ decays faster or slower for a larger or smaller $\Gamma$ [compare the decay rates of Fig. 9(a), for $\Gamma=1 \mathrm{kHz}$, with those of Fig. 9(b), for $\Gamma=10 \mathrm{kHz}$, the fixed frequency oscillation can be observed (left insets) as a key DTC feature for tens or hundreds of Floquet cycles on the millisecond scale. It is also clear that $|P(n)|$ typically suffers a faster decay for a larger $L$. For illustrative purposes, we fit our numerical data for the curves of $|P(n)|$ shown in Fig. 9(a) 
using a single exponential decay for $n>75:|P(n)| \sim e^{-\alpha n}$, thus obtaining the decay rate $\alpha$ plotted in the right inset of Fig. $9\left(a_{2}\right)$ as a function of $L$. We note, however, that the curves for a larger, and more realistic, $\Gamma$ shown in Fig. 9(b) show a more complex behavior.

Similar results to those just discussed on the effects of the finite lifetime of the Rydberg level are also obtained for nonvanishing values of $\Delta$ and for both models above (Secs. II and IV).

\section{CONCLUSION}

In summary, we carried out extensive numerical experiments on two realistic models exhibiting DTC features based on a ring of cold Rydberg atoms interacting via van der Waals potentials. Our results show that, by applying suitable sequences of driving fields with a Floquet periodicity $T$, it is viable to fulfill all three conditions required for DTCs. The population difference oscillates with a period $2 T$ (discrete time-translational symmetry breaking), such a period is robust against parameter variations (rigidity), the oscillations remain in phase over an increasingly long time when the chain length increases (persistence). Employing an exact diagonalization approach, we can deal with chain sizes of up to 14 atoms, which may definitely be of experimental interest. We characterized the parameter ranges amenable to the observation of the DTC regime for two distinct setups. While these share many common features, such as the symmetry for a simultaneous sign change of both the driving detuning $\Delta$ and the dipole-dipole interaction $V$, they are differently sensitive to the value of $\Delta$. Finally, we considered the effects of a finite lifetime of the Rydberg level which may limit the persistence of the DTC features for realistic values of $\Gamma$. In this regards, however, we stress that all other parameters considered here are widely tunable: the Rabi frequency $\Omega$ and detuning $\Delta$ being directly controlled via the intensity and frequency of the driving laser fields, while the interaction parameter $V$ by the distance between the adjacent potential wells in the chain. Thus, the models we consider here are amenable to experimental verification even in the case in which extrinsic effects, such as noise associated to the laser stability or other dephasing effects, might effectively lead to larger values of $\Gamma$.

\section{ACKNOWLEDGMENTS}

The work is supported by the National Natural Science Foundation of China (Grants No. 11534002 and No. 11674049) as well as the Cooperative Program by the Italian Ministry of Foreign Affairs and International Cooperation (Grant No. PGR00960) and the National Natural Science Foundation of China (Grant No. 11861131001).

\section{APPENDIX A: NUMERICAL APPROACH}

To study the emergence of the DTC features in our Rydberg-chain system, we resorted to an exactdiagonalization (ED) approach. Below we comment on the applicability of other routinely employed numerical methods to probe the Floquet dynamics of quantum many-body systems.

Let us start from purely Hamiltonian situations (i.e., we neglect dissipation for a moment). For the sake of clarity, here we explicitly refer to the model of Sec. II; however, from a computational point of view, the employed procedure and the complexity of calculations are totally equivalent in the other considered models. Our simulations make use of the full $2^{L}$-dimensional Hilbert space of the system, with $L$ being the number of Rydberg atoms in the simulated chain. We start from a given initial state $|\Psi(0)\rangle$ in such a space, written in the basis $\left\{|g\rangle_{j},|r\rangle_{j}\right\}_{j=1, \ldots, L}$, and repeatedly apply the Floquet time-evolution operator

$$
U_{F}(1)=U_{2} U_{1}=e^{-i H_{2} T_{2} / \hbar} e^{-i H_{1} T_{1} / \hbar}
$$

as in Eq. (3). While $U_{2}$ is already diagonal in the above computational basis, and thus can be trivially applied to a generic input state with $O\left(2^{L}\right)$ operations, the evaluation of the nondiagonal operator $U_{1}$ generally requires the full diagonalization of the matrix $H_{1}$ in Eq. (1). Unfortunately, besides the exponential growth of the system's Hilbert space, the need to calculate and manipulate all the eigenvalues and eigenvectors of $H_{1}$ requires to act on $O\left(2^{2 L}\right)$ complex elements, thus severely limiting present-day numerical capabilities up to $L \sim 15$ atoms.

In principle, other less computationally demanding, yet approximate, methods could be used to track the quantum dynamics of the system. For example, one could either resort to a numerical integration of the Schrödinger equation governing the time evolution of the system (either via a standard RungeKutta approach or with a Suzuki-Trotter decomposition of the unitary evolution operator), or to techniques based on the density-matrix renormalization group (DMRG). The first type of approaches still requires manipulating and keeping track of the full $2^{L}$-dimensional Hilbert space, although the full Hamiltonian spectrum is not explicitly used (taking advantage of the sparseness of the various Hamiltonian matrices, one would be able to deal with sizes of $L \sim 30$ atoms). However, we checked that the need to reach very long times, up to several thousands of Floquet time intervals (see, e.g., Figs. 4 and 6 where $n T \gtrsim 0.1 \mathrm{~s}$ ) to monitor the persistence properties of the DTC phase, limits the applicability of approximate methods, due to accumulation of numerical errors. Likewise, the entanglement growth along the Floquet time evolution prevents DMRG-based algorithms to achieve such regimes, although DTC signatures for a relatively small number of Floquet periods and much larger systems have been reported in a similar context [31].

Finally, we briefly discuss the simulation procedure adopted for a nonunitary dynamics in the presence of losses, as described by the master equation (8). Even in this case we employed an ED method, allowing to reach arbitrarily large Floquet cycles without error accumulation. To this purpose, we first vectorize the system's density matrix

$$
\left.\rho=\sum_{i, j} \rho_{i, j}|i\rangle\langle\boldsymbol{j}|\rightarrow| \rho\rangle\right\rangle \equiv \sum_{i, j} \rho_{i, j}|\boldsymbol{i}\rangle \otimes|\boldsymbol{j}\rangle,
$$

with $\boldsymbol{i} \equiv\left\{i_{1}, \ldots, i_{L}\right\}$ (and $i_{\ell}$ denoting the state of the $\ell$ th Rydberg atom), and then formally write the master equation as a linear differential equation on a vectorized state of $2^{2 L}$ components (analogously to the Schrödinger equation, which acts on a pure state of $2^{L}$ components):

$$
\left.\left.\partial_{t}|\rho\rangle\right\rangle=\mathcal{L}(t)|\rho\rangle\right\rangle .
$$


Here $\mathcal{L}(t)$ denotes the Liouvillian superoperator, of size $2^{2 L} \times$ $2^{2 L}$, applied to the vectorized state $\left.|\rho\rangle\right\rangle$, and obtained from the master equation through the same mapping as in Eq. (A1). Similarly as for the Hamiltonian case, the ED approach deals with the full Liouvillian spectrum, thus acting on $O\left(2^{4 L}\right)$ complex elements; for the times we were interested in, we were able to reach systems with up to $L=10$ sites.

\section{APPENDIX B: FEW-BODIES AND FEW-CYCLES DYNAMICS}

In this Appendix, we show explicit analytical results on $P(n)$ in the few-cycles and few-bodies regime, with the purpose to gain a qualitative understanding of the dependence on parameters of the DTC features shown in Figs. 4, 5, 6, and 7. Let us introduce a simplified model, for which it is possible to derive an analytic solution in a relatively compact form, and set $V=0$ during $T_{1}$ such that only Rabi flipping and detuning terms are admitted in the first stage of the Floquet cycle. The corresponding Hamiltonian is

$$
\tilde{H}_{1}=\hbar \sum_{j=1}^{L}\left[\Omega\left(\sigma_{j}^{+}+\sigma_{j}^{-}\right)+\Delta N_{j}^{r}\right] .
$$

On the other hand, both detuning and interaction terms are present in the second stage, which is thus described by the Hamiltonian $\mathrm{H}_{2}$ in Eq. (2).

In the case of two atoms, the stroboscopic evolution operator $\widetilde{U}_{F}=U_{2} \widetilde{U}_{1}$ is obtained by composing the following explicit matrix representation:

$$
\widetilde{U}_{1}=\left(\begin{array}{cccc}
X_{+}^{2} e^{i 2 \theta_{1}} & i X_{+} Y e^{i 2 \theta_{1}} & i X_{+} Y e^{i 2 \theta_{1}} & -Y^{2} e^{i 2 \theta_{1}} \\
i X_{+} Y & X^{2} & -Y^{2} & i X_{-} Y \\
i X_{+} Y & -Y^{2} & X^{2} & i X_{-} Y \\
-Y^{2} e^{-i 2 \theta_{1}} & i X_{-} Y e^{-i 2 \theta_{1}} & i X_{-} Y e^{-i 2 \theta_{1}} & X_{-}^{2} e^{-i 2 \theta_{1}}
\end{array}\right)
$$

and

$$
U_{2}=\left(\begin{array}{cccc}
1 & 0 & 0 & 0 \\
0 & e^{-i \theta_{2}} & 0 & 0 \\
0 & 0 & e^{-i \theta_{2}} & 0 \\
0 & 0 & 0 & e^{-i\left(2 \theta_{2}+\theta_{3}\right)}
\end{array}\right)
$$

where

$$
\begin{aligned}
X_{ \pm} & =\cos \left(\Omega_{e} T_{1}\right) \pm i\left(\Delta / \Omega_{e}\right) \sin \left(\Omega_{e} T_{1}\right) \\
Y & =\left(\Omega / \Omega_{e}\right) \sin \left(\Omega_{e} T_{1}\right) \\
X & =\sqrt{X_{+} X_{-}}, \\
\theta_{1} & =\Delta T_{1} / 2, \quad \theta_{2}=\Delta T_{2}, \quad \text { and } \theta_{3}=V T_{2} .
\end{aligned}
$$

The operator $\widetilde{U}_{F}$ acts on the $4 \times 1$ column vector obtained by arranging the two-atom state basis $|g g\rangle,|g r\rangle,|r g\rangle$, and $|r r\rangle$ in this order. Thus,

$$
|\Psi(n)\rangle=\widetilde{U}_{F}(n)|\Psi(0)\rangle=\left[U_{2} \widetilde{U}_{1}\right]^{n}|\Psi(0)\rangle .
$$

Assuming that the atomic evolution starts from the ground state, i.e., that its components on the basis used in the above matrix representations (B2) and (B3) are $\Psi_{1}(0)=1$ and $\Psi_{2}(0)=\Psi_{3}(0)=\Psi_{4}(0)=0$, we then have $P(0)=-1$ with $P(n)=\Psi_{4}^{*}(n) \Psi_{4}(n)-\Psi_{1}^{*}(n) \Psi_{1}(n)$. After two Floquet cycles, we obtain

$$
\begin{aligned}
P(2)= & -X^{8}+2 X^{4} Y^{4}-Y^{8}+\left[2 X_{+}^{2} Y^{4}\left(Y^{2}+X^{2}\right) e^{i\left(\varphi_{1}+\theta_{3}\right)}\right. \\
& \left.+2 X_{+}^{2} X^{2} Y^{2}\left(Y^{2}+X^{2}\right) e^{i \varphi_{1}}+\text { c.c. }\right]
\end{aligned}
$$

where $\varphi_{1}=2 \theta_{1}+\theta_{2}$. After the third cycle, we have

$$
\begin{aligned}
P(3)= & -X^{12}-5 X^{8} Y^{4}-16 X^{6} Y^{6}-11 X^{4} Y^{8}+Y^{12}+\left[2 X_{+}^{6} Y^{4}\left(X^{2}+Y^{2}\right) e^{i\left(3 \varphi_{1}+2 \theta_{3}\right)}+2 X_{+}^{4} Y^{4}\left(X^{4}-Y^{4}\right) e^{i\left(2 \varphi_{1}+2 \theta_{3}\right)}\right. \\
& -2 X_{+}^{6} Y^{4}\left(X^{2}+Y^{2}\right) e^{i\left(3 \varphi_{1}+\theta_{3}\right)}+4 X_{+}^{4} Y^{4}\left(X^{4}+2 X^{2} Y^{2}+Y^{4}\right) e^{i\left(2 \varphi_{1}+\theta_{3}\right)}+2 X_{+}^{2} Y^{4}\left(4 X^{6}-6 X^{4} Y^{2}+9 X^{2} Y^{4}-Y^{6}\right) e^{i\left(\varphi_{1}+\theta_{3}\right)} \\
& +2 X_{+}^{4} X^{2} Y^{2}\left(X^{4}-Y^{4}\right) e^{2 i \varphi_{1}}+2 X_{+}^{2} Y^{2}\left(2 X^{8}+8 X^{6} Y^{2}-10 X^{4} Y^{4}+7 X^{2} Y^{6}-Y^{10}\right) e^{i \varphi_{1}} \\
& \left.+2 X^{2} Y^{4}\left(X^{6}+X^{4} Y^{2}-X^{2} Y^{4}-Y^{6}\right) e^{i \theta_{3}}+\text { c.c. }\right],
\end{aligned}
$$

which is sensitive to $\varphi_{1}$ (that is, to $\Delta$ ) and $\theta_{3}$ (that is, to $V$ ) in a more complicated way than $P(2$ ).

In an analogous way, in the case of three atoms for which $\widetilde{U}_{1}$ and $U_{2}$ become $8 \times 8$ matrices, we obtain

$$
\begin{aligned}
P(2)= & -\left(X^{2}-Y^{2}\right)^{2}\left(Y^{2}+X^{2}\right)^{4}+\left[2 X_{+}^{4} Y^{6}\left(2 X^{2}-Y^{2}\right) e^{i\left(2 \varphi_{1}+3 \theta_{3}\right)}+2 X_{+}^{2} Y^{6}\left(Y^{2}+X^{2}\right)^{2} e^{i\left(\varphi_{1}+2 \theta_{3}\right)}\right. \\
& \left.+4 X_{+}^{2} X^{2} Y^{4}\left(Y^{2}+X^{2}\right) e^{i\left(\varphi_{1}+\theta_{3}\right)}+2 X_{+}^{2} X^{4} Y^{2}\left(Y^{2}+X^{2}\right)^{2} e^{i \varphi_{1}}+\text { c.c. }\right] .
\end{aligned}
$$

If the signs of both $\Delta$ and $V$ are changed, $X$ and $Y$ are invariant while $X_{+}$and $X_{-}$exchange roles, while the phases $\varphi_{1}$ and $\theta_{3}$ and their linear combinations change signs. As in all the above expressions for $P(n)$ all complex terms are summed with their complex conjugate ones, $P(n)$ remains the same. In other terms, the contributions to $P(n)$ contained in the square brackets of the above equations reduce to a sum of terms of the form

$$
f_{1}\left(\Delta^{2 j}\right) \cos \left[g_{1}(\Delta, V)\right]+f_{2}\left(\Delta^{2 j-1}\right) \sin \left[g_{2}(\Delta, V)\right]
$$

with $f_{1,2}$ and $g_{1,2}$ denoting certain linear functions, and thus $P(n)$ is invariant when the signs of $\Delta$ and $V$ are simultaneously changed [as in Figs. 5( $\left.c_{1}\right), 5\left(c_{2}\right), 7\left(c_{1}\right)$, and $\left.7\left(c_{2}\right)\right]$.

We also note that the interference phase terms involve increasingly varied combinations of $\varphi_{1}=\Delta\left(T_{1}+T_{2}\right)$ and $\theta_{3}=V T_{2}$ as the Floquet cycle $n$ and/or the chain size of $L$ increase. The number of such linear combinations grows exponentially as $2^{L-1} 4^{n-2}$ for $L \geqslant 2$ Rydberg atoms and $n \geqslant$ 2 Floquet cycles, while the oscillations of $X_{ \pm}$and $Y$ only depend on the phase $\alpha=\Omega_{e} T_{1}$. It is to be noticed that in the 
model of Sec. II the value of $\varphi_{1}$ is much more sensitive to $\Delta$ than is $\alpha$ as $T_{2} \gg T_{1}$, while this is not the case for the model of Sec. IV.

We also note that the interference phase terms involve increasingly varied combinations of $\varphi_{1}=\Delta\left(T_{1}+T_{2}\right)$ and $\theta_{3}=$ $V T_{2}$ as the Floquet cycle $n$ and/or the chain size $L$ increase. The number of such linear combinations grows exponentially as $2^{L-1} 4^{n-2}$ for $L \geqslant 2$ Rydberg atoms and $n \geqslant 2$ Floquet cycles, which constitutes an essential ingredient for realizing the expected DTC features. We further note that $n_{c}$ in the model of Sec. II exhibits sharp discrete structures with the peak amplitudes decaying faster for larger $|\Delta|$ due to the effectively reduced atom-field coupling strength. In particular, the period is $0.457 \mathrm{MHz}$ for $\epsilon=0.4 \mathrm{MHz}$ in Fig. $5\left(\mathrm{c}_{1}\right)$ while $0.366 \mathrm{MHz}$ for $\epsilon=-0.4 \mathrm{MHz}$ in Fig. 5( $\left.\mathrm{c}_{2}\right)$, which are, respectively, larger and smaller than $2 \pi /\left(T_{1}+T_{2}\right)=0.393 \mathrm{MHz}$, a period determined by fast oscillating phase $\varphi_{1}$ with $T_{2} \gg T_{1}$. The opposite perturbation effects of positive and negative $\epsilon$ on this intermediate period should arise from slow oscillating phase $\Omega_{e} T_{1}$ in $X_{ \pm}$and $Y$, in which $\epsilon$ and $\Delta$ exhibit a complex interplay sensitive to the sign of $\epsilon$. It is thus natural to expect vanishing discrete structures [see Figs. $7\left(c_{1}\right.$ and $7\left(c_{2}\right)$ ] in the model of Sec. IV where $\varphi_{1}=\Delta T_{1}$ becomes a slow oscillating phase.
[1] F. Strocchi, Symmetry Breaking, Lecture Notes in Physics Vol. 732 (Springer, Berlin, 2008).

[2] F. Wilczek, Phys. Rev. Lett. 109, 160401 (2012).

[3] P. Bruno, Phys. Rev. Lett. 111, 070402 (2013).

[4] H. Watanabe and M. Oshikawa, Phys. Rev. Lett. 114, 251603 (2015).

[5] C. Heissenberg and F. Strocchi, arXiv:1906.12293.

[6] V. K. Kozin and O. Kyriienko, Phys. Rev. Lett. 123, 210602 (2019).

[7] K. Sacha, Phys. Rev. A 91, 033617 (2015).

[8] V. Khemani, A. Lazarides, R. Moessner, and S. L. Sondhi, Phys. Rev. Lett. 116, 250401 (2016).

[9] D. V. Else, B. Bauer, and C. Nayak, Phys. Rev. Lett. 117, 090402 (2016).

[10] N. Y. Yao, A. C. Potter, I.-D. Potirniche, and A. Vishwanath, Phys. Rev. Lett. 118, 030401 (2017).

[11] V. Khemani, C. W. von Keyserlingk, and S. L. Sondhi, Phys. Rev. B 96, 115127 (2017).

[12] J. Zhang, P. W. Hess, A. Kyprianidis, P. Becker, A. Lee, J. Smith, G. Pagano, I.-D. Potirniche, A. C. Potter, A. Vishwanath, N. Y. Yao, and C. Monroe, Nature 543, 217 (2017).

[13] S. Choi, J. Choi, R. Landig, G. Kucsko, H. Z. Zhou, J. Isoya, F. Jelezko, S. Onoda, H. Sumiya, V. Khemani, C. V. Keyserlingk, N. Y. Yao, E. Demler, and M. D. Lukin, Nature 543, 221 (2017).

[14] S. Autti, V. B. Eltsov, and G. E. Volovik, Phys. Rev. Lett. 120, 215301 (2018).

[15] J. Smits, L. Liao, H. T. C. Stoof, and P. van der Straten, Phys. Rev. Lett. 121, 185301 (2018).

[16] S. Pal, N. Nishad, T. S. Mahesh, and G. J. Sreejith, Phys. Rev. Lett. 120, 180602 (2018).

[17] J. Rovny, R. L. Blum, and S. E. Barrett, Phys. Rev. Lett. 120, 180603 (2018).

[18] W. W. Ho, S. Choi, M. D. Lukin, and D. A. Abanin, Phys. Rev. Lett. 119, 010602 (2017).

[19] D. V. Else, B. Bauer, and C. Nayak, Phys. Rev. X 7, 011026 (2017).

[20] T.-S. Zeng and D. N. Sheng, Phys. Rev. B 96, 094202 (2017).

[21] F. Iemini, A. Russomanno, J. Keeling, M. Schirò, M. Dalmonte, and R. Fazio, Phys. Rev. Lett. 121, 035301 (2018).

[22] Z. Gong, R. Hamazaki, and M. Ueda, Phys. Rev. Lett. 120, 040404 (2018).

[23] B. Zhu, J. Marino, N. Y. Yao, M. D. Lukin, and E. A. Demler, New J. Phys. 21, 073028 (2019).
[24] P. Matus and K. Sacha, Phys. Rev. A 99, 033626 (2019).

[25] E. Lustig, Y. Sharabi, and M. Segev, Optica 5, 1390 (2018).

[26] K. Giergiel, A. Dauphin, M. Lewenstein, J. Zakrzewski, and K. Sacha, New J. Phys. 21, 052003 (2019).

[27] K. Sacha and J. Zakrzewski, Rep. Prog. Phys. 81, 016401 (2018).

[28] D. V. Else, C. Monroe, C. Nayak, and N. Y. Yao, arXiv:1905.13232.

[29] D. M. Basko, I. L. Aleiner, and B. L. Altschuler, Ann. Phys. (NY) 321, 1126 (2006).

[30] R. Nandkishore and D. A. Huse, Annu. Rev. Condens. Matter Phys. 6, 15 (2015).

[31] B. Huang, Y. H. Wu, and W. V. Liu, Phys. Rev. Lett. 120, 110603 (2018).

[32] A. Russomanno, F. Iemini, M. Dalmonte, and R. Fazio, Phys. Rev. B 95, 214307 (2017).

[33] W. C. Yu, J. Tangpanitanon, A. W. Glaetzle, D. Jaksch, and D. G. Angelakis, Phys. Rev. A 99, 033618 (2019).

[34] F. M. Gambetta, F. Carollo, M. Marcuzzi, J. P. Garrahan, and I. Lesanovsky, Phys. Rev. Lett. 122, 015701 (2019).

[35] F. M. Surace, A. Russomanno, M. Dalmonte, A. Silva, R. Fazio, and F. Iemini, Phys. Rev. B 99, 104303 (2019).

[36] R. E. Barfknecht, S. E. Rasmussen, A. Foerster, and N. T. Zinner, Phys. Rev. B 99, 144304 (2019).

[37] D. A. Abanin, W. De Roeck, W. W. Ho, and F. Huveneers, Phys. Rev. B 95, 014112 (2017).

[38] A. Lazarides and R. Moessner, Phys. Rev. B 95, 195135 (2017).

[39] I. Bloch, J. Dalibard, and S. Nascimbene, Nat. Phys. 8, 267 (2012)

[40] H. Labuhn, D. Barredo, S. Ravets, S. de Léséleuc., T. Macrì, T. Lahaye, and A. Browaeys, Nature 534, 667 (2016).

[41] H. Kim, W. Lee, H. Lee, H. Jo, Y. Song, and J. Ahn, Nat. Commun. 7, 13317 (2016).

[42] D. Barredo, S. de Léséleuc, V. Lienhard, T. Lahaye, and A. Browaeys, Science 354, 1021 (2016).

[43] H. Bernien, S. Schwartz, A. Keesling, H. Levine, A. Omran, H. Pichler, S. Choi, A. S. Zibrov, M. Endres, M. Greiner, V. Vuletić, and M. D. Lukin, Nature 551, 579 (2017).

[44] T. Gallagher, Rydberg Atoms (Cambridge University Press, Cambridge, England, 2005).

[45] M. Saffman, T. G. Walker, and K. Mølmer, Rev. Mod. Phys. 82, 2313 (2010). 
[46] T. Amthor, C. Giese, C. S. Hofmann, and M. Weidemüller, Phys. Rev. Lett. 104, 013001 (2010).

[47] D. Yan, Y. M. Liu, Q. Q. Bao, C. B. Fu, and J. H. Wu, Phys. Rev. A 86, 023828 (2012).

[48] Y. M. Liu, D. Yan, X. D. Tian, C. L. Cui, and J. H. Wu, Phys. Rev. A 89, 033839 (2014).

[49] C. J. Turner, A. A. Michailidis, D. A. Abanin, M. Serbyn, and Z. Papic, Nat. Phys. 14, 745 (2018).

[50] C. H. Fan, H. X. Zhang, and J. H. Wu, Phys. Rev. A 99, 033813 (2019).

[51] L. Isenhower, E. Urban, X. L. Zhang, A. T. Gill, T. Henage, T. A. Johnson, T. G. Walker, and M. Saffman, Phys. Rev. Lett. 104, 010503 (2010).

[52] I. E. Protsenko, G. Reymond, N. Schlosser, and P. Grangier, Phys. Rev. A 65, 052301 (2002).

[53] T. Keating, R. L. Cook, A. M. Hankin, Y. Y. Jau, G. W. Biedermann, and I. H. Deutsch, Phys. Rev. A 91, 012337 (2015).

[54] X. D. Tian, Y. M. Liu, C. L. Cui, and J. H. Wu, Phys. Rev. A 92, 063411 (2015).

[55] D. D. Bhaktavatsala Rao and K. Mølmer, Phys. Rev. Lett. 111, 033606 (2013).

[56] M. Saffman and T. G. Walker, Phys. Rev. A 66, 065403 (2002).
[57] Y. M. Liu, X. D. Tian, X. Wang, D. Yan, and J. H. Wu, Opt. Lett. 41, 408 (2016).

[58] J.-H. Wu, M. Artoni, F. Cataliotti, and G. C. La Rocca, Europhys. Lett. 120, 54002 (2018).

[59] I. D. Potirniche, A. C. Potter, M. Schleier-Smith, A. Vishwanath, and N. Y. Yao, Phys. Rev. Lett. 119, 123601 (2017).

[60] O. Morsch and I. Lesanovsky, Riv. Nuovo Cimento 41, 383 (2018).

[61] A. Browaeys, D. Barredo, and T. Lahaye, J. Phys. B: At. Mol. Opt. Phys. 49, 152001 (2016).

[62] A. F. Linskens, I. Holleman, N. Dam, and J. Reuss, Phys. Rev. A 54, 4854 (1996).

[63] C. Cohen-Tannoudji, J. Dupont-Roc, and G. Grynberg, AtomPhoton Interactions (Wiley, New York, 1998).

[64] N. B. Delone and V. P. Krainov, Phys. Usp. 42, 669 (1999).

[65] J. A. Gordon, C. L. Holloway, A. Schwarzkopf, D. A. Anderson, S. Miller, N. Thaicharoen, and G. Raithel, Appl. Phys. Lett. 105, 024104 (2014).

[66] L. Hao, Y. Xue, J. Fan, Y. Jiao, J. Zhao, and S. Jia, Appl. Sci. 9, 1720 (2019).

[67] C. Gardiner and P. Zoller, Quantum Noise (Springer, Berlin, 2004). 